\title{
From Parallel Virtual Machine to Virtual Parallel Machine: The Unibus System
}

\author{
Vaidy Sunderam \\ Department of Mathematics and Computer Science \\ Emory University \\ Atlanta, Georgia, USA \\ vss@emory.edu
}

Network-based concurrent computing frameworks have matured over two decades. However, in the realm of multi-domain geographically distributed computing, their true potential is yet to be realized. Popularly termed "grids", these metacomputing systems have yet to see widespread adoption and use, partly due to challenges resulting from heterogeneity, deployment issues, and dynamicity. The Unibus project proposes novel strategies for unifying and dynamically conditioning shared heterogeneous resources located within multiple administrative domains. Our approach is based on client-centric overlay software that provides unified interfaces to diverse resources, complemented by runtime systems that substantially automate setup and configuration. The overlay unifies heterogeneous resources through service drivers or mediators that operate analogously to device drivers. New resources and variations in availability are handled through self-deployment of mediators and their service daemon counterparts, enabling the overlay to adaptively present applications with coherent aggregated projections of the underlying resources. Dynamic incorporation of new resources is facilitated via automated environment conditioning that uses deployment descriptors in conjunction with site-specific data to prepare platforms for execution. We will discuss the motivations and rationale behind the Unibus approach, describe the design of the framework, and present preliminary results and experiences. 